\title{
Constituents of Erythrina - a Potential Source of Secondary Metabolities: A Review
}

\author{
Mohammad Musarraf Hussain', Md. Tariqul Haque Tuhin², Fahima Akter \\ and Mohammad A. Rashid ${ }^{3}$
}

\author{
${ }^{1}$ Department of Pharmacy, Jagannath University, Dhaka-1100, Bangladesh \\ ${ }^{2}$ Department of Pharmacy, State University of Bangladesh, Dhaka-1205, Bangladesh \\ ${ }^{3}$ Department of Pharmaceutical Chemistry, University of Dhaka, Dhaka-1000, Bangladesh
}

Received: February 09, 2016; Accepted: March 20, 2016; Published (Web): July 31, 2016

\begin{abstract}
The genus Erythrina is a potential source of chemical constituents, many of which medicinal properties. Although some reviews on chemical constituents of particular Erythrina species could be found, no detailed review covering the chemistry of different Erythrina species has been reported to the best of my knowledge. Therefore, the aim of this review was to compile the phytoconstituents reported from various species of Erythrina. A total of 155 secondary metabolites have been published from 15 species of Erythrina. Among them $E$. subumbrans and E. variegata consist of the highest number of chemical constituents.
\end{abstract}

Key words: Erythrina, Phytoconstituents, Alkaloids, Flavonoids, Pterocarpans, Steroids, Triterpenes

\section{Introduction}

The genus Erythrina (Leguminosae) consists of 110 species of trees and shrubs. Among them, 15 different species have been thoroughly analyzed in this review. E. addisoniae is a wild tree, small to medium sized and contains good quantity of potassium salts in its fruits. E. americana is a $5 \mathrm{~m}$ tall tree and its branches are widely spread. E. caribaea and E. indica are medium sized tree, normally grow 6 - $9 \mathrm{~m}$ in height and its leaves are trifoliate, bright-emerald green. $E$. latissima is $9-24 \mathrm{~m}$ in height with root and stem burnt (Wanjala et al., 2002). E. melanacantha has smaller leaves and shorter, standard calyx (Gillett, 1972). E. mildbraedii grows upto $30 \mathrm{~m}$ in height and is native in West Africa. E. poeppigiana is a roadside tree having 8 - $12 \mathrm{~m}$ height with orange colored flowers (Tanaka et al., 2003). E. stricta Roxb is a midsize tree with cracked cork bark having pale yellow prickle branches (Hussain et al., 2011). E. subumbrans is a deciduous, medium sized tree (5 - $25 \mathrm{~m}$ tall). Three leaflets are present in leaves at alternate arrangement and its bark is whitish. E. variegata is a first growing deciduous tree with 15-18 $\mathrm{m}$ tall and leaves are 6 inches long having spiny branches (Kumar et al., 2010). E. vespertilio is an ambiguous tree (common name: bean tree) and indigenous to North Australia. E. velutina is a leguminous tall tree that grows upto $10 \mathrm{~m}$ and indigenous to Brazil.

E. zeberi and E. zeyheri are deciduous subshurb trees growing upto $60 \mathrm{~cm}$ height having glabrous, trifoliolate leaves with large leaflets armed. The species of Erythrina have been used as traditional medicaments as sedative, antiasthmatic, antiepileptic, anticonvulsant, antipyretic, antiinflammation, antibacterial, insomnia, helminthiasis, cough, cuts and wounds (Kumar et al., 2010).

Although some reviews carring chemical constituents of particular Erythrina species have been found, no detailed review was found on different Erythrina species. Thus, this paper will assist the researchers working on Erythrina species around the globe.

\section{Phytoconstituents}

A total of 15 species of Erythrina have been analyzed and 155 (1-155) molecules were reported in this review as phytoconstituents. The species are $E$.

Correspondence to: Mohammad Musarraf Hussain; Email: m.musarraf.hussain@gmail.com 
addisoniae, E. caribaea, E. indica, E. lattisima, E. melanacantha, E. mildbraedii, E. poeppigiana, E. stricta, E. subumbrans, E. veriagata, E. vespertilio, E. velutina, E. zeberi, E. zeyheri and E. americana. Different chemical constituents such as - alkaloids, flavonoids, pterocarpans, triterpenes and steroids were extracted from these compounds. Erythrina is a prominent source of alkaloid. A bunch of alkaloids are isolated from these fifteen species of Erythrina such as - Erysovine- $N$-oxide (1), Erysosalvinone (2), Erysodine (3), $1 \mathrm{H}$-indole-3-propanamide (4), Glucoerysodine (5), Erysotrine (6), Erysovine (7), Erythraline (8), Erythramine (9), Erysopine (10), Erythrocarine (11), Erythrinine (17), 10, 11-Dioxyerysotrine (34), Erysosalvine (41), Erymelanthine (43), Melanacanthine (43), 8-Oxa- $\alpha$-erythroidine (54), 8-Oxo- $\alpha$-erythroidine epoxide (61), 8-Oxo- $\alpha$-erythroidine (62), 8-Oxoerythraline epoxide (63), 11-Hydroxyepierythratidine (66), Erythrinan (67), 11-Acetryl erysotrine (68), Erythratidinone (69), 10,11-dioxo- erythrartine (79), 10,11-dioxoepierythrstidine (80), 10,11-erythratidinone (81), Epierythratine (94), 11-Hydroxyerythratine (95),
11-Hydroxyepierythratine (96), Erythritol (99), Isococolinine (103), Erythratidinone (106), Demethoxyerythratidinone (107), Erythramine (108), Erysopitine (109), Erysodienone (110), 11Methoxyerythraline (141) and E-erythsotin (142), Sodium erysovine $15-O$-sulfate (143), Erysopine 15- $O$ sulfate (144), 16- $O-\beta$-D-Glucopyranosyl coccoline (145), Sodium erysovine $N$-oxy-15- $O$-sulfate (146), 11 Oxoerythraline (147), $\beta$-erythroidine (153), Dihydro- $\beta$ erythroidine (154) and Wilsonine (155) (Figure 1) (Amer et al., 1991; Boland et al., 1998; Cui et al., 2009; Faria et al., 2007; Garin-Aguilar et al., 2000; Haggins et al., 1981; Hauschild et al., 2010; Hussain, 2002; Jang et al, 2008; Kabenei et al., 2011; Kumar et al., 2010; Soto-Hernadez et al., 2012; Lundquist, 1973; Miyazawa et al, 2006; Nakayama et al, 1978; Ozawa et al., 2011; Rodriguez et al., 2004; Watjen et al., 2008; Rahman et al., 2007; Rahman et al., 2010; Rodriguez et al., 2004; Rukachaisirikul et al., 2007; Rukachaisirikul et al., 2008; Soto-Hernadez et al, 2012; Tanaka et al., 2001; Tanaka et al., 2003; Wanjala et al, 2002; Zheng et al., 2013; Zhou et al., 2011).<smiles>COc1cc2c(cc1O)[C@@]13C[C@H](OC)C=CC1=CCN3CC2</smiles>

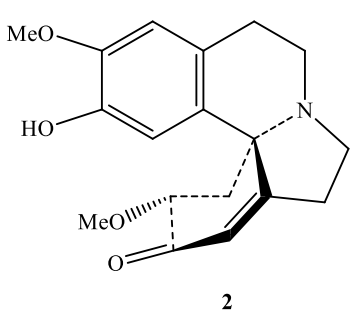

2

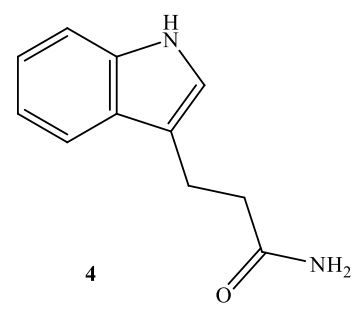<smiles>COc1cc2c(cc1O)[C@@]13C[C@@H](OC)C=CC1=CCN3CC2</smiles>
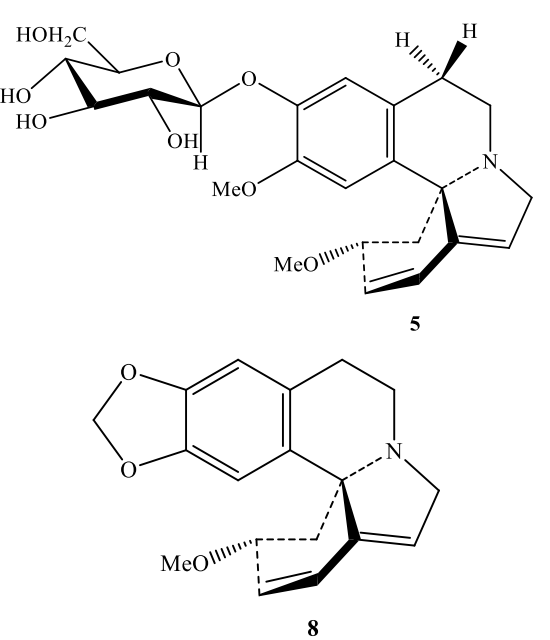<smiles>COc1cc2c(cc1O)CCN1CC=C3C=C[C@@H](OC)C[C@]321</smiles>

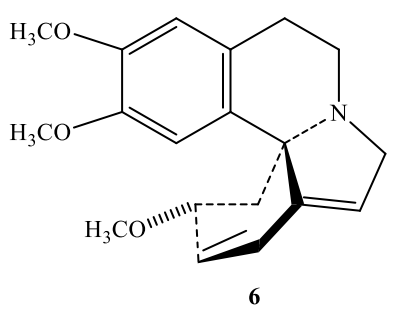

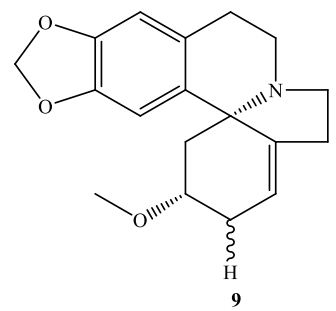


<smiles>CO[C@H]1C=C[C@@H]2CCN3CCc4cc(O)c(O)cc4[C@@]23C1</smiles><smiles>O[C@H]1C=C[C@H]2CCN3CCc4cc5c(cc4[C@]23CC1)OCO5</smiles><smiles>CO[C@H]1C=CC2=CCC3CC(O)c4cc5c(cc4C23C1)OCO5</smiles><smiles>COc1cc2c(cc1OC)[C@]13CCN1C(=O)C(=O)c1ccc(cc1)CC23</smiles>

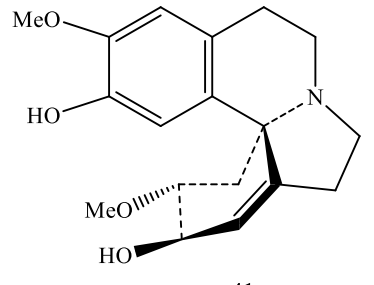

41<smiles>COC1C=CC2=CC(=O)N3CCC4COC(=O)C=C4[C@]23C1</smiles>

61

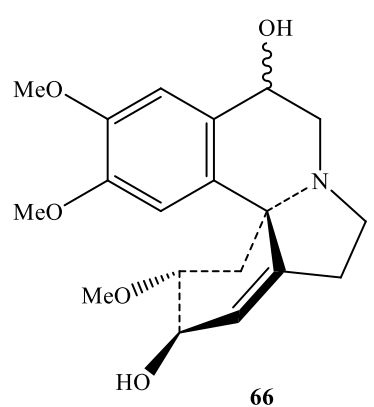<smiles>CC(=O)COC(=O)C1CC2=CC(=O)C3(C1)c1cc(C(C)=O)c(OC(C)=O)cc1CCN3CC2</smiles><smiles>C1=CC2CCN3CCc4ccccc4C23CC1</smiles>

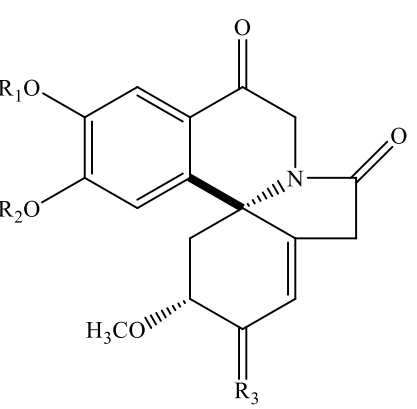

79: $\mathrm{R}_{1}+\mathrm{R}_{2}=\mathrm{CH}_{2}, \mathrm{R}_{3}=$ Beta- $\mathrm{OH}, \mathrm{H}$ 80: $\mathrm{R}_{1}=\mathrm{R}_{2}=\mathrm{CH}_{3}, \mathrm{R}_{3}=$ Beta-OH, $\mathrm{H}$ 81: $\mathrm{R}_{1}=\mathrm{R}_{2}=\mathrm{CH}_{3}, \mathrm{R}_{3}=\mathrm{O}$<smiles>CC(=O)c1cc2c(cn1)CCN1C(=O)C=C3C=CC=CC321</smiles>

43<smiles></smiles>

63<smiles>CC(=O)OC(C)=O</smiles>

68

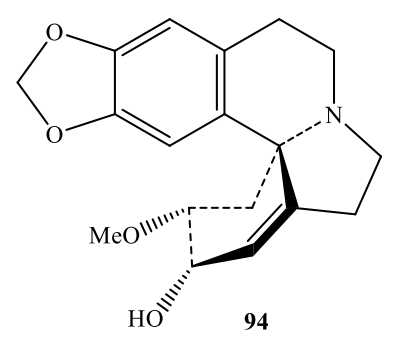




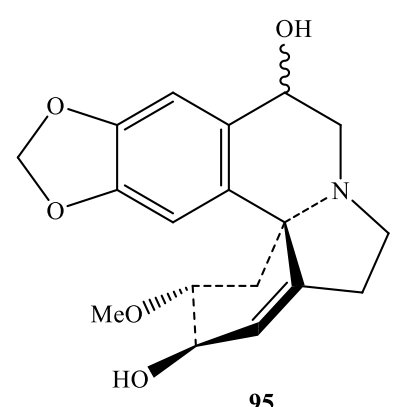

95<smiles>COc1cc2c(cc1OC)C13CC(OC)C=CC1=CC(=O)N3CC2</smiles>

103

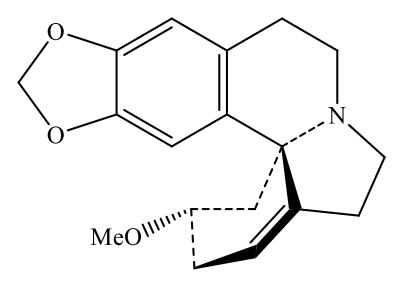

108

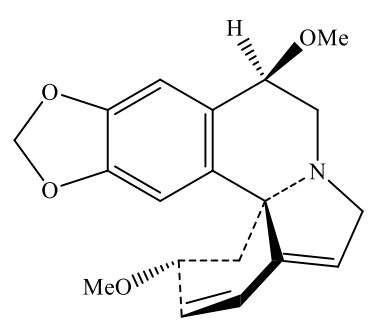

141<smiles></smiles>
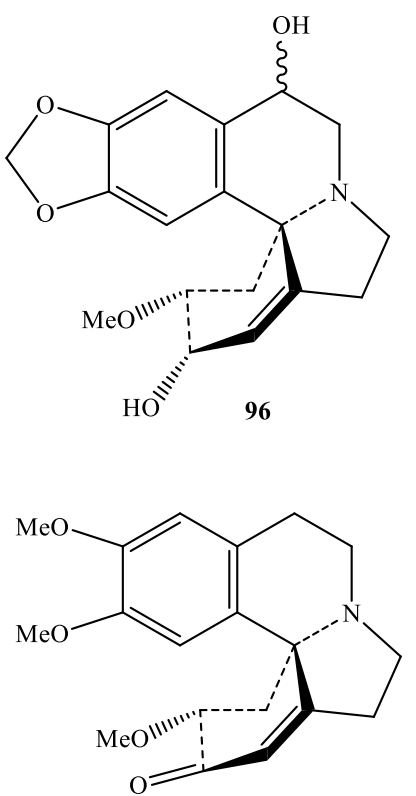

106

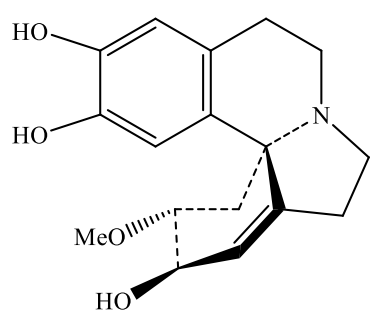

109

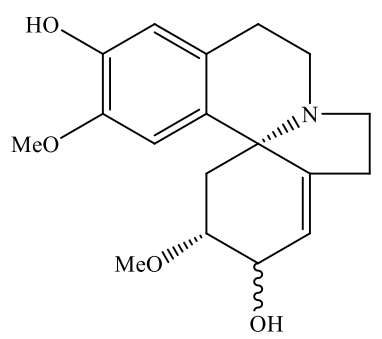

142<smiles>COC1=CC23C(=CC1O)CCN2CCc1cc(OC)c(OC)cc13</smiles>

99<smiles>COc1cc2c(cc1OC)[C@@]13CC[I-]C(=O)C=C1CCN3CC2</smiles>

107

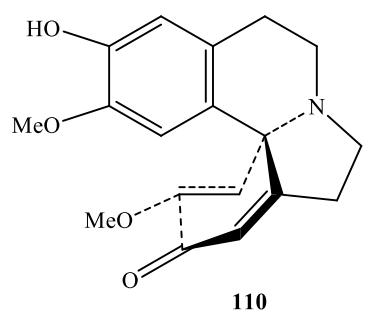

110<smiles>[R20]c1cc2c(cc1[R20])[C@]13CC(OC)C=CC1=CC([X])N3CC2</smiles>

143: $\mathrm{R}_{1}=\mathrm{Me}, \mathrm{R}_{2}=\mathrm{SO}_{3} \mathrm{Na}, \mathrm{X}=\mathrm{H}_{2}$

144: $\mathrm{R}_{1}=\mathrm{H}, \mathrm{R}_{2}=\mathrm{SO}_{3} \mathrm{H}, \mathrm{X}=\mathrm{H}_{2}$

145: $R_{1}=$ Beta-D-Glc, $R_{2}=M e, X=O$<smiles>CO[C@@H]1C=CC2=CCN3CCC4=C(CC(=O)OC4)[C@]23C1</smiles>

153 
<smiles>CO[C@H]1C=CC2CCN3CCC4=C(CC(=O)OC4)[C@]23C1</smiles>

154

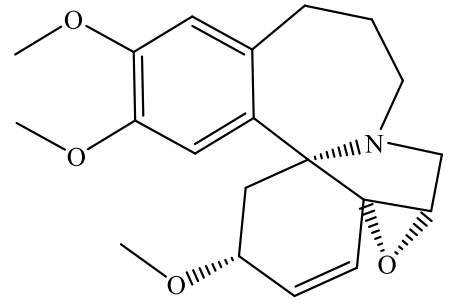

155

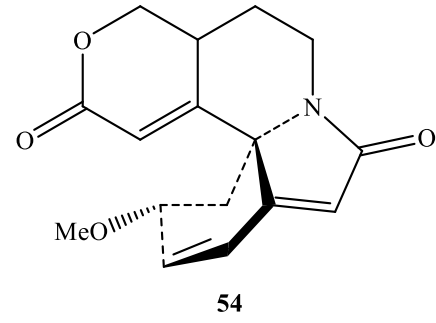

54

Figure 1. Alkaloids from different species of Erythrina.

Many falvonoids have been derived from these 15 species of Erythrina such as - Indicanine A (12), Indicanine B (13), Indicanine C (14), Indicanine D (15), Indicanine E (16), Wighteone (18), Alpinumisoflavone (19), Dimethylalpinumisoflavone (20), Erythrinin C (21), Erysenegalensein E (22), Abyssinone IV (28), Erylatissin A (29), Erylatissin B (30), Erylatissin C (31), Erythrinin B (33), Neobavaisoflavone (37), Licoflavone-4'-O-methyl ether (44), 2',7-Dihydroxy4'-methoxy-5'-(3-methylbut-2-enyl)isoflavone (45), (3R)-2',7-Dihydroxy-3'-(3-methylbut-2-enyl)-2"', 2"' dimethylpyrano $\left[5^{\prime \prime \prime}, 6^{\prime \prime \prime}, 4^{\prime}, 5^{\prime}\right] \quad$ isoflavan (46), Abyssinin II (47), Parvisoflavone B (48), Erypoegin G (50), Erysubin F (57), 5-Hydroxysophoranone (75), Abyssinone V (78), Lespedezaflavanone B (87), Glabrol (91), Vogelin C (93), Erythrinins A (100), 6Hydroxygenistein (101), Eryvarin B (111), Eryvarin F (112), Eryvarin M (114), Eryvarin N (115), Eryvarin O (116), Scandenone (123), 5,7,4'-trihydroxy-6,8diprenylisoflavone (124), 4',5,7-Trihydroxy-8prenylisoflavone (125), 4',5,7-Trihydroxy-8methylisoflavone (126), Isobavachin (128), 5,4'Dihydroxy-8-(3,3-dimethylallyl)-2"'-methoxyisopropyl furano[4,5:6,7]isoflavone (132), 5,7,4'-Trihydroxy-6(3,3- dimethylallyloxiranylmethyl) isoflavone (133), 5,4'-Dihydroxy-8-(3,3-dimethylallyl)-2"'-hydroxymethyl-2"-methylpyrano[5,6:6,7] isoflavone (134), 5,4'-Dihydroxy-2'-methoxy-8-(3,3-dimethyl-allyl)2",2"-dimethylpyrano[5,6:6,7] isoflavone (135), Euchrenone $b_{10}$ (136), Isoerysenegalensein E (137), Laburnetin (138), Lupiwighteone (139), Eryzerin A (148), Eryzerin B (149), Eryzerin C (150), Eryzerin D (151) (Figure 2) (Boland et al., 1998; Chacha et al., 2005; Hussain et al., 2008; Hussain et al., 2011; Jang et al., 2008; Kobayashi et al., 1997; Koo et al., 2013; Kumar et al., 2010; Lundquist, 1973; Miyazawa et al., 2006; Nakayama et al., 1978; Nkengfack et al., 2001; Rahman et al., 2007; Rahman et al., 2010; Rodriguez et al., 2004; Rukachaisirikul et al., 2007; Rukachaisirikul et al., 2007; Sato et al., 2003; SotoHernadez et al., 2012; Talikepali et al., 1990; Tanaka et al., 2001; Tanaka et al., 2002; Tanaka et al., 2003; Tanaka et al., 2004; Tanaka et al, 2011; Tchokouaha et al., 2010; Waffo et al., 2000; Wanjala et al., 2002; Watjen et al., 2008; Xiaoli et al., 2006; Zheng et al., 2013; Zhou et al., 2011).
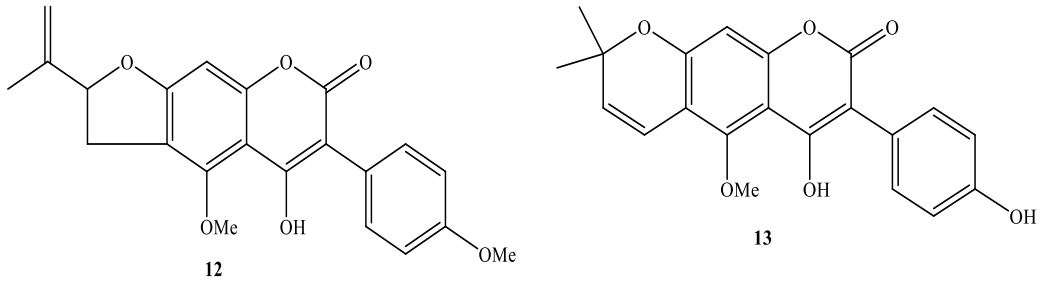

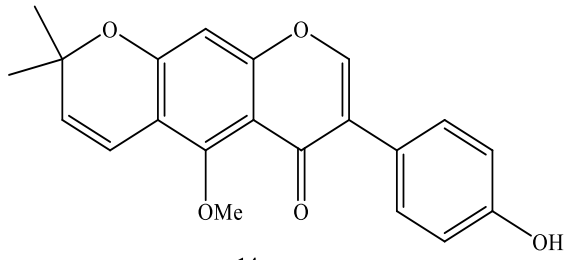

14 
242

Hussain et al. / Bangladesh Pharmaceutical Journal 19(2): 237-253, 2016<smiles>C=C(C)C(O)Cc1c(O)c(CC=C(C)C)c(Cl)c2c(=O)c(-c3ccc(CC(C)C(C)C)cc3)coc12</smiles><smiles>CC1(C)C=Cc2c(cc3occ(-c4ccc(O)cc4)c(=O)c3c2O)O1</smiles>

19
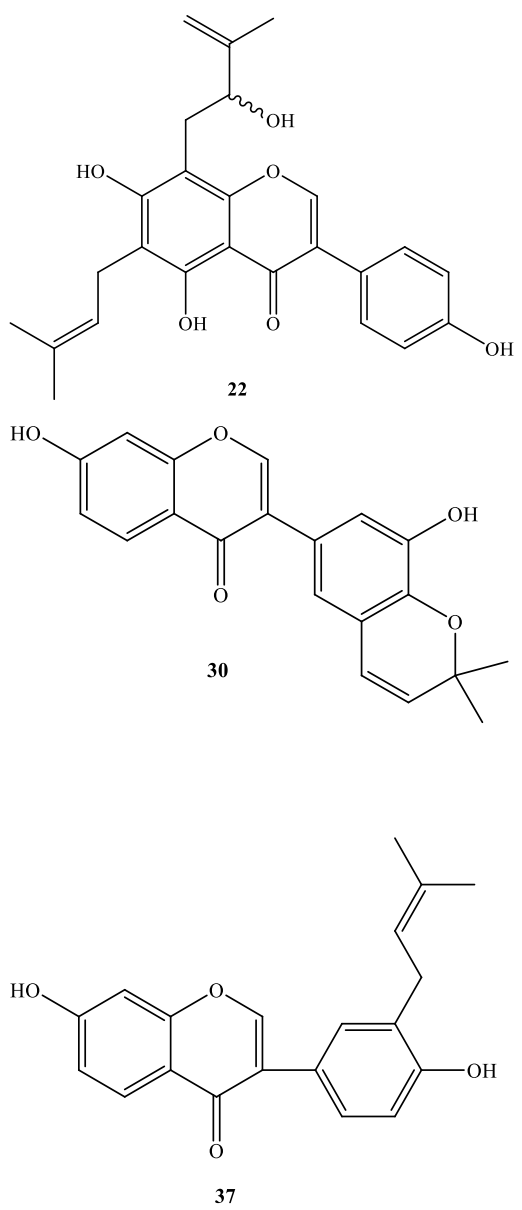<smiles>COc1ccc(-c2coc3cc4c(c(OC)c3c2=O)C=CC(C)(C)O4)c(O)c1</smiles><smiles>COc1ccc(-c2coc3cc4c(c(OC)c3c2=O)C=CC(C)(C)O4)cc1</smiles>

20
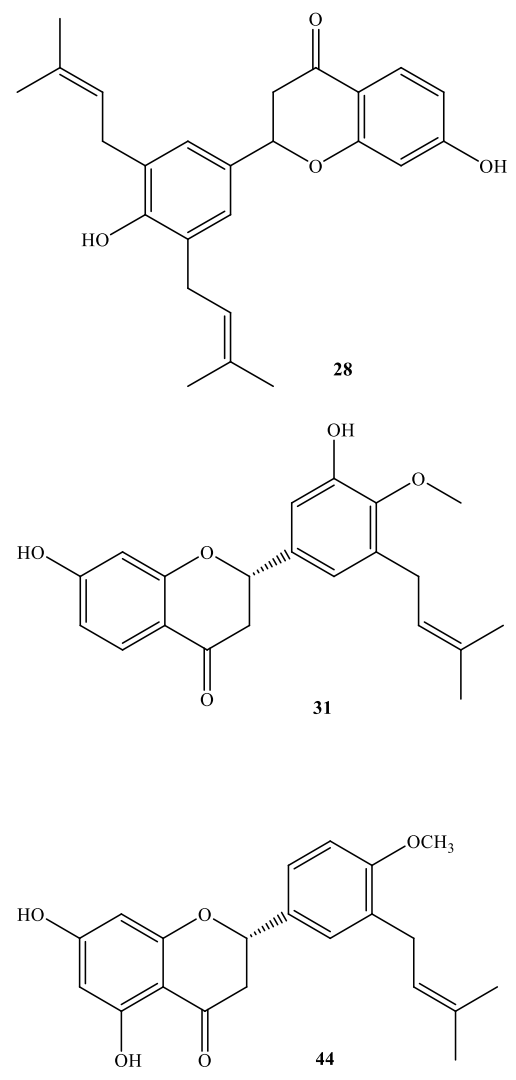

45
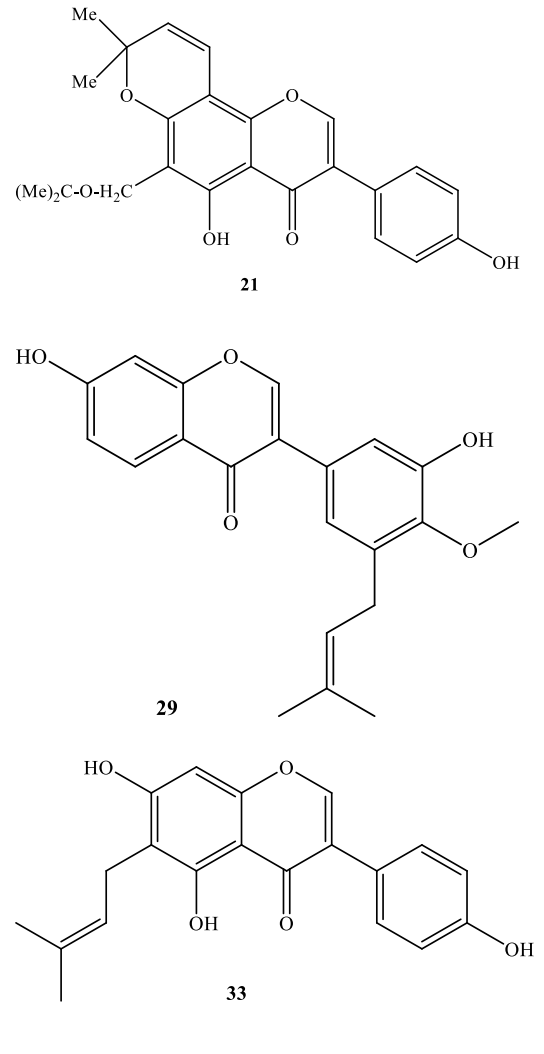<smiles>COc1cc(O)c(-c2coc3cc(O)ccc3c2=O)cc1CC=C(C)C</smiles> 
HO<smiles>CC(C)=CCc1c(O)c([C@H]2COc3cc(Cl)ccc3C2)cc2c1OC(C)(C)C=C2</smiles><smiles>COc1cc(O)c2c(c1)OCC(c1cc3c(cc1OC)OC(C)(C)C=C3)C2=O</smiles>

50<smiles>CC(C)=CCc1cc(C2CC(=O)c3c(O)cc(O)cc3O2)cc(CC=C(C)C)c1O</smiles><smiles>CC(C)=CCc1c(O)cc(O)cc1-c1cc(=O)c2c(O)cc(O)c(CC=C(C)C)c2o1</smiles><smiles>CC(C)=CCc1c(O)c2c(c3c(=O)c(-c4ccc(O)cc4)coc13)OC(C)(C)[C@H](O)C2</smiles><smiles>CC(C)=CCc1cc(-c2coc3c(CC=C(C)C)c(O)ccc3c2=O)ccc1O</smiles><smiles>COc1cc([C@H]2CC(=O)c3c(O)cc(O)cc3O2)cc(CC=C(C)C)c1O</smiles><smiles>CC1(C)C=Cc2c(cc3occ(-c4ccc(O)cc4O)c(=O)c3c2O)O1</smiles>

48
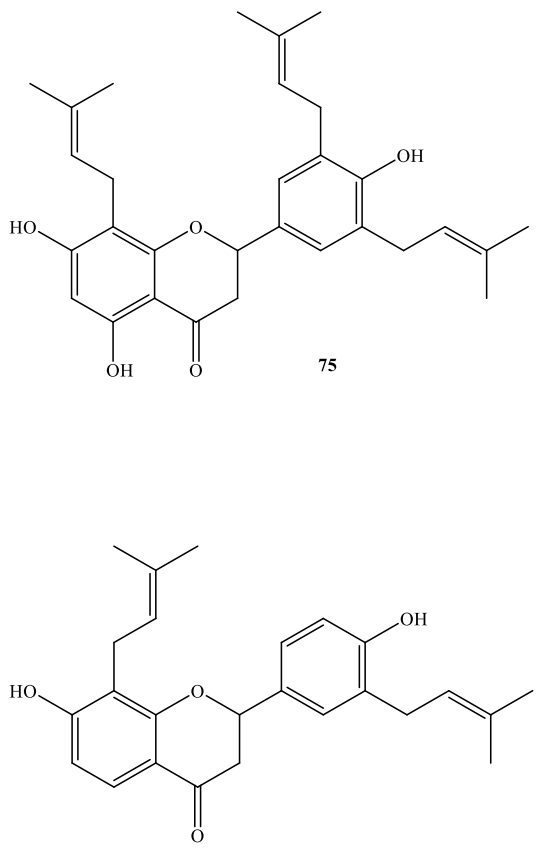

91

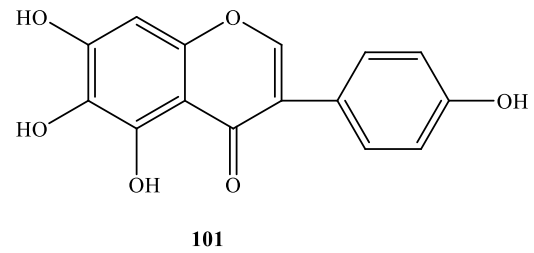<smiles>COc1cc(C2COc3cc(C)ccc3C2=O)c(OC)cc1O</smiles><smiles>COc1c(-c2cc3ccc(O)cc3oc2=O)ccc(O)c1CC=C(C)C</smiles> 

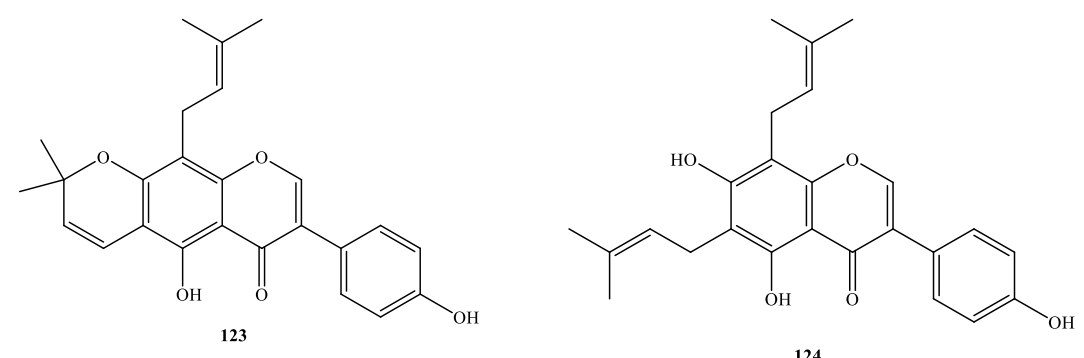

124

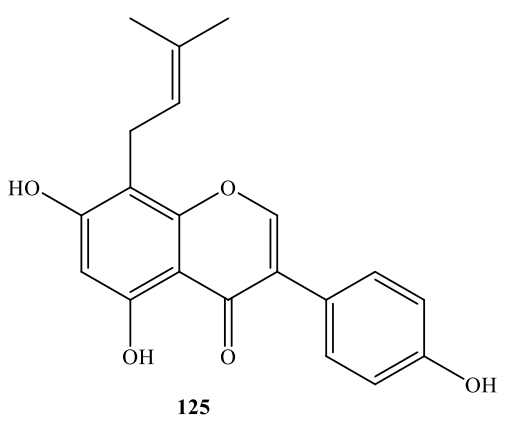

125<smiles>Cc1c(O)cc(O)c2c(=O)c(-c3ccc(O)cc3)coc12</smiles><smiles>COC(C)(C)c1cc2c(O)c3c(=O)c(-c4ccc(O)cc4)coc3c(CC=C(C)C)c2o1</smiles><smiles>COc1cc(O)ccc1-c1coc2cc(O)c(CC3OC3(C)C)c(O)c2c1=O</smiles>

134<smiles>CC(C)=CCc1c2c(c3occ(-c4ccc(O)cc4)c(=O)c3c1O)CC(C(C)(C)O)O2</smiles><smiles>CC(C)=CCc1c(O)ccc2c1O[C@H](c1ccc(O)cc1)CC2=O</smiles>

128<smiles>CC(C)=CCc1c2c(c(O)c3c(=O)c(-c4ccc(O)cc4)coc13)C=CC(C)(C(=O)O)O2</smiles><smiles>COc1cc(O)ccc1C1COc2c(CC=C(C)C)c3c(c(O)c2C1=O)C=CC(C)(C)O3</smiles><smiles>C=C(C)C(O)Cc1c(O)c(CC=C(C)C)c2occ(-c3ccc(O)cc3)c(=O)c2c1O</smiles> 
<smiles>C=C(C)C(O)Cc1c(O)cc2occ(-c3ccc(O)cc3)c(=O)c2c1O</smiles>

138<smiles>CC(C)=CCc1c(O)cc(O)c2c(=O)c(-c3ccc(Cl)cc3)coc12</smiles><smiles>CC(C)=CCc1ccc([C@H]2COc3c(cc(O)c(CC=C(C)C)c3CC=C(C)C)CO2)c(O)c1CC=C(C)C</smiles><smiles>COc1cc(O)ccc1[C@H]1COc2c(cc(CC=C(C)C)c(O)c2CC=C(C)C)C1=O</smiles><smiles>CCOc1ccc(C2COc3c(cc4c(c3CC=C(C)C)OC(C)(C)C=C4)C2)c(O)c1</smiles><smiles>CC(C)=CCc1cc2c(=O)c(Oc3ccc(O)cc3O)coc2c(CC=C(C)C)c1O</smiles><smiles>COc1cc(C2COc3c(ccc(O)c3CC=C(C)C)C2=O)c(OC)cc1O</smiles>

Figure 2. Flavonoids reported from Erythrina species.

The plant belonging to the genus Erythrina contain a number of pterocarpans such as - Erybraedin A (32), 2-(5'-Hydroxy-3-methoxyphenyl)-6-hydroxy-5-methoxybenzofuran (35), Isoneorautenol (38), Erymelanthine (42), Erypoegin F (49), Erypoegin H (51), Erypoegin I (52), Erypoegin J (53), Cristacaprin (55), Dimethylmedicaprin (56), Eryvarin D (58), Folitenol
(59), Erystagallin C (64), Eryvarin A (65), Erystagallin A (76), Erycristagallin (77), 1-Methoxyerythrabissin (82), Erythrabissin-I (83), Erythrabissin-II (84), Erybraedin B (85), Hydroxycristacarpone (86), Phaseollin (89), Eryvarin E (90), Dihydrofolinin (97), Orientanol-B (104), Phaseollidin (127) and Eryzerins E (152) (Figure 3) (Amir et al., 2011; Boland et al., 1998; 
Hauschild et al., 2010; Innok et al., 2010; Kabenei et al., 2011; Kobayashi et al., 1997; Lundquist, 1973; Miyazawa et al., 2006; Soto-Hernadez et al., 2012; Rahman et al., 2007; Rukachaisirikul et al., 2007;
Rukachaisirikul et al., 2008; Tanaka et al., 1996; Tanaka et al., 2002; Tanaka et al., 2002; Zheng et al., 2013; Zhou et al., 2011).<smiles>CC(C)=CCc1c(O)ccc2c1OC1c3ccc(O)c(CC=C(C)C)c3OCC21</smiles>

HO<smiles>COc1ccc2c(C=O)c(-c3ccc(Cl)cc3O)oc2c1CC=C(C)C</smiles>

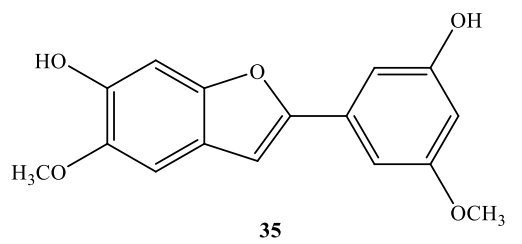

35<smiles>CC1(C)C=Cc2cc3c(cc2O1)OC1c2ccc(O)cc2OCC31</smiles>

38
$\mathrm{HO}$<smiles>CC(C)=CCc1c(O)ccc2c3c(oc12)-c1ccc(C)cc1OC3</smiles>

$\mathrm{HO}$<smiles>COc1ccc2c(c1CC(=O)C(C)C)O[C@H]1c3ccc(O)cc3OC[C@]21O</smiles><smiles>COc1ccc2c(c1CC=C(C)C)O[C@H]1c3ccc(O)cc3OC[C@]21O</smiles><smiles>[CH2][C@@]1(O)COc2cc(OC(C)(C)C=C)c1cc2[C@@H]1Oc2c1ccc(OC)c2CC=C(C)C</smiles>

HO<smiles>Oc1ccc2c(c1)OC1c3ccc(O)cc3OCC21</smiles> 

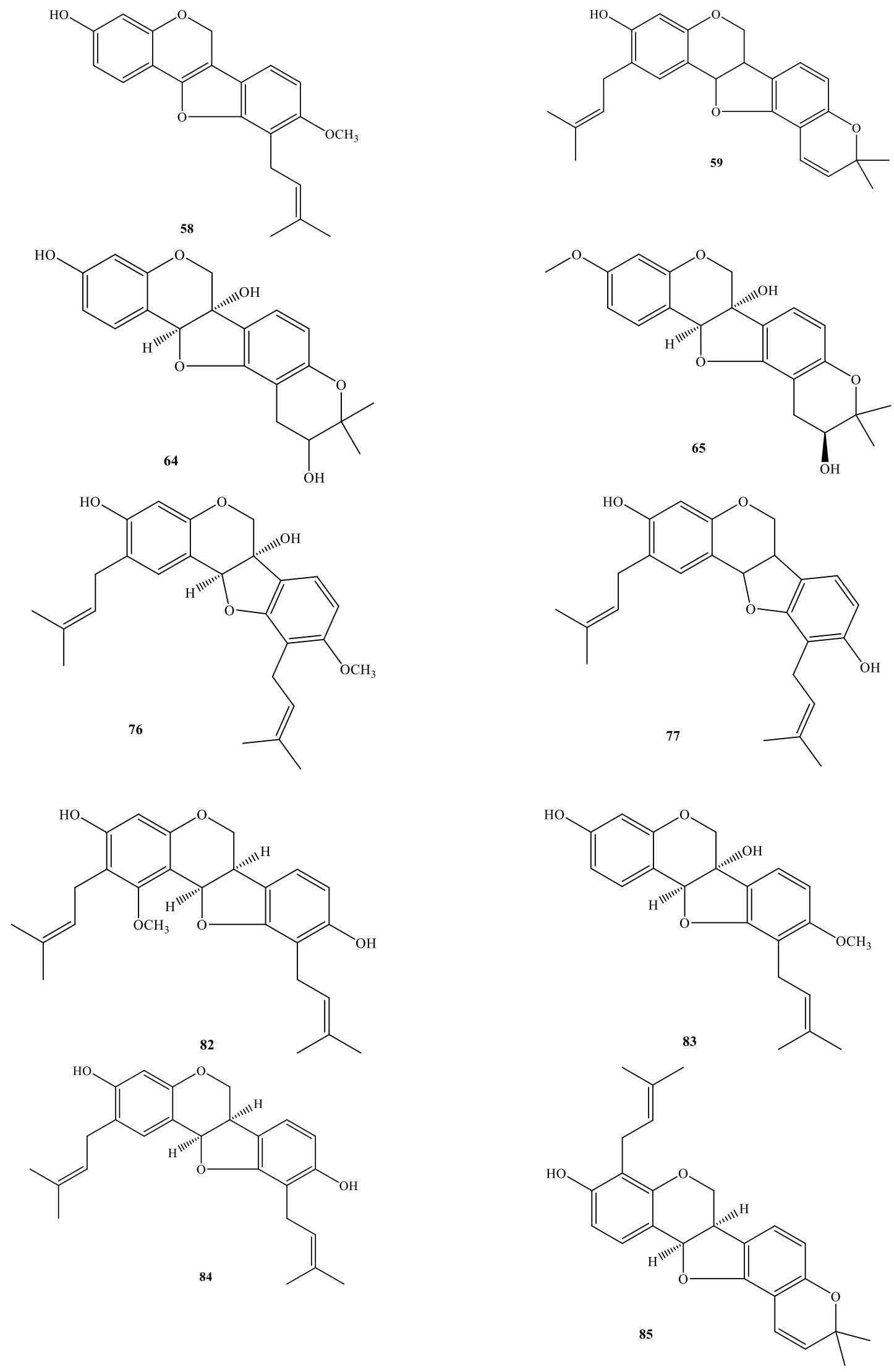
<smiles>COc1ccc2c(c1CC=C(C)C)O[C@H]1C[C@]3(O)C=CC(=O)C=C3O[C@]21O</smiles><smiles>COc1ccc2c3c(oc2c1CC=C(C)C)-c1cc(CC=C(C)C)c(O)cc1OC3</smiles><smiles>COc1cc2c(cc1CC=C(C)C)[C@H]1Oc3cc(O)ccc3[C@H]1CO2</smiles>

104<smiles>CC1(C)C=Cc2c(ccc3c2O[C@H]2c4ccc(O)cc4OC[C@H]32)O1</smiles><smiles>CC1(C)CCc2cc3c(cc2O1)OCC1c2ccc4c(c2OC31)CCC(C)(C)O4</smiles><smiles>CC(C)=CCc1c(O)ccc2c1O[C@H]1c3ccc(O)cc3OC[C@H]21</smiles><smiles>COc1ccc2c(c1CC=C(C)C)O[C@H]1c3ccc(O)c(CC=C(C)C)c3OC[C@]21O</smiles>

Figure 3. Pterocarpans from fifteen species of Erythrina.

Erithrina species contain some triterpenes and steroids. These are - Oleanolic acid (24), Erythrodiol (25), Stigmasterol (26), Sophoradiol (70), Stigmasta-4en-3-one (71), Stigmasta-4,22-dien-3-one (72), 3 $\beta$ hydroxystigmasta-5,22-dien-7-one (73), Melilotigenin C (74), Lupeol (88), Soyasapogenol B (92), Epilupeol
(98) and 3 $\beta$-28-dihydroxyolean-12-ene (102) (Figure 4) (Amir et al., 2011; Boland et al., 1998; Hauschild et. al., 2010; Kobayashi et al., 1997; Lundquist, 1973; Miyazawa et al., 2006; Soto-Hernadez et al., 2012; Tanaka et al., 2002; Zheng et al., 2013; Zhou et al., 2011). 


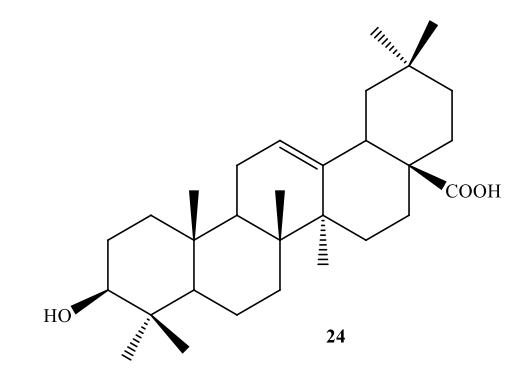

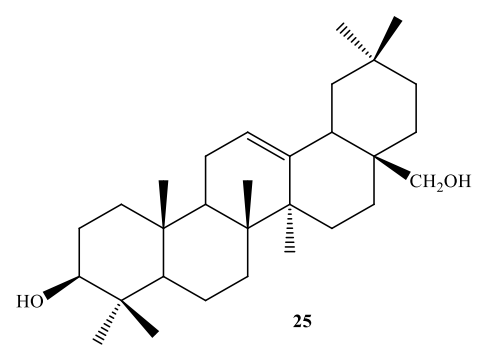<smiles>CCC(/C=C/C(C)C1CCC2C3CC=C4CC(C)CCC4(C)C3CCC12C)C(C)C</smiles><smiles>CC1(C)CC2C3=CCC4[C@@]5(C)CC[C@H](O)C(C)(C)C5CC[C@@]4(C)[C@]3(C)CC[C@@]2(C)[C@@H](O)C1</smiles><smiles>CC[C@H](CC[C@@H](C)C1CCC2[C@]1(C)CC[C@@]1(C)C3(C)CCC(=O)C=C3CC[C@]21C)C(C)C</smiles><smiles>CCC(/C=C/C(C)C1CCC2(C)C3CCC4=CC(=O)CCC4(C)C3CCCC12C)C(C)C</smiles><smiles>CCC(/C=C/[C@@H](C)[C@H]1CCC2C3C(=O)C=C4C[C@@H](O)CC[C@]4(C)C3CC[C@]21C)C(C)C</smiles>

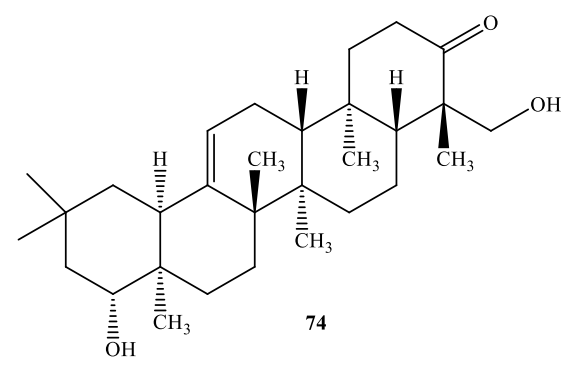<smiles>[CH][C@@H]1CC(C)(C)C2CCC3(C)C(CCC4C5C(C(=C)C)CCC5(C)CCC43C)C2(C)C1</smiles>

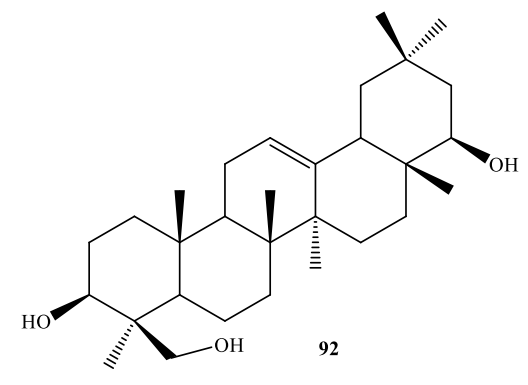

88<smiles>C=C(C)C1CCC2(C)CCC3(C)C(CCC4C5(C)CC[C@H](I)C(C)(C)C5CCC43C)C12</smiles><smiles>CC1(C)CCC2(C=O)CCC3(C)C(=CCC4C5(C)CCC(O)C(C)(C)C5CCC43C)C2C1</smiles>

Figure 4. Triterpenes and steroids from different species of Erythrina.

A good number of miscellaneous classes of compounds were also extracted from these fifteen species of Erythrina, including Erythrinassinate B (23), 5-O- $\beta$-D-xylo-pyranoside (27), Neorautenol
(36), 
Syringaresinol (39), Vanillic acid (40), Orientanol C (60), Octacosyl ferulate (105), Eryvarin G (113), Eryvarin P (117), Eryvarin Q (118), Eryvarin R (119), Eryvarin V (120), Eryvarin W (121), Eryvarin X (122), Eryvaiestyrene (129), Eryvarinol A (130), Eryvarinol B
(131), Eryvarin H (140) (Figure 5) (Amir et al., 2011; Boland et al., 1998; Koo et al., 2013; Lundquist, 1973; Miyazawa et al., 2006; Rahman et al., 2007; SotoHernadez et al., 2012; Zheng et al., 2013; Zhou et al., 2011).<smiles>CCCCCCCCCCCCCCCCCCCOC(=O)/C=C/c1ccc(O)c(OCCCCCCCCCC)c1</smiles><smiles>COc1cc([C@@H]2OC[C@]3(C)[C@@H](c4cc(OC)c(O)c(OC)c4)OC[C@H]23)cc(O)c1O</smiles><smiles></smiles><smiles>CC(C)=CCc1c2c(cc3c(=O)c(Oc4ccc(O)cc4O)coc13)C=CC(C)(C)O2</smiles>
113<smiles>COc1cc(O)c(CC=C(C)C)cc1-c1oc2c(CC=C(C)C)c(O)ccc2c1C#N</smiles>
118<smiles>O=C(O)c1cc(OC2OCC(O)C(O)C2O)ccc1O</smiles><smiles>CC1(C)C=Cc2cc3c(cc2O1)OCC1c2ccc(O)cc2OC31</smiles><smiles>COc1cc(C(=O)O)ccc1O</smiles>

40<smiles>CCOC(=O)CCc1ccc(OC)c(O)c1</smiles><smiles>COc1cc(-c2oc3cc(O)ccc3c2C=O)c(OC)cc1O</smiles><smiles>COc1cc(C2(C)COc3cc(O)ccc32)c(OC)cc1O</smiles> 

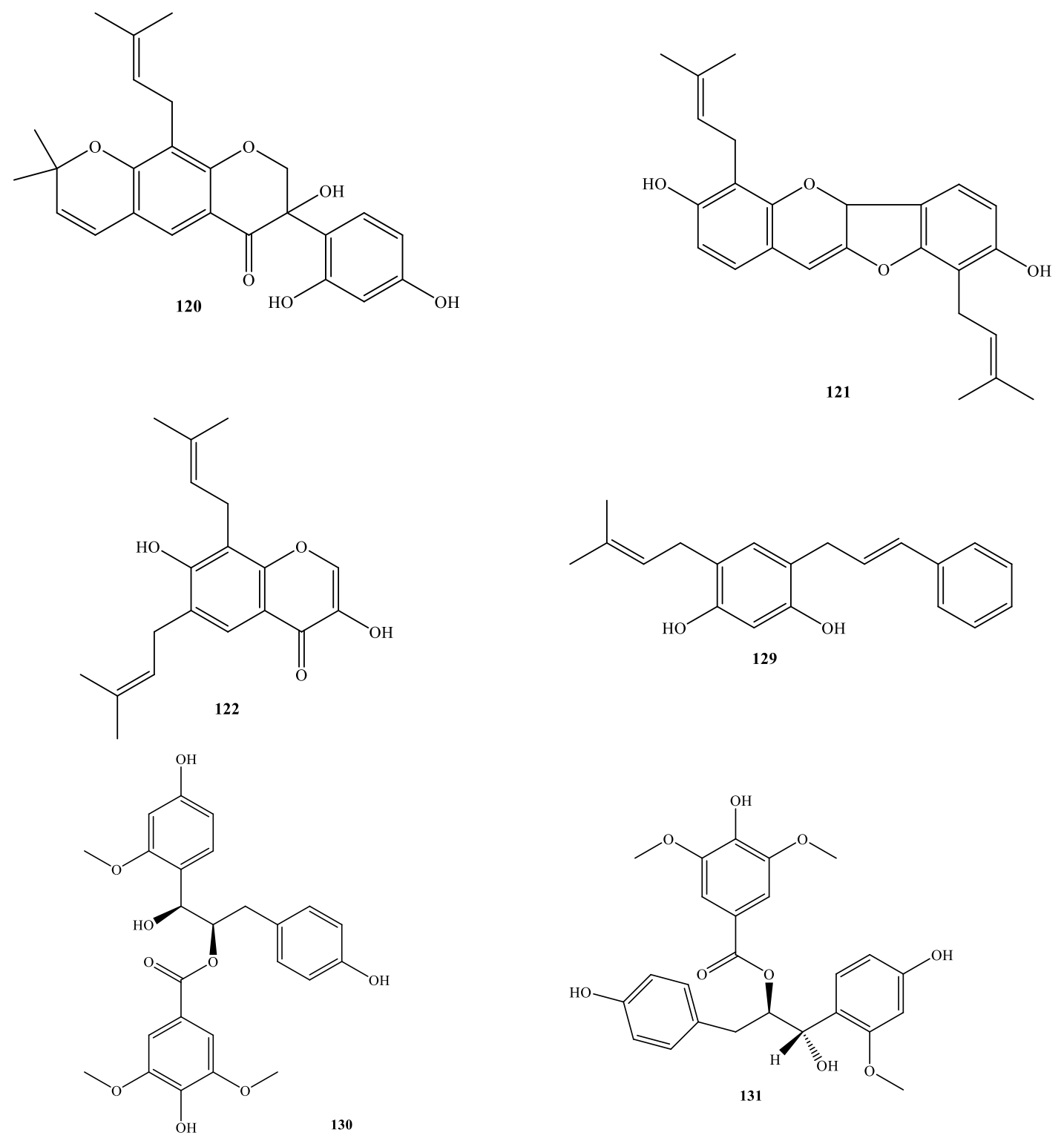<smiles>COc1cc(C2=Cc3ccc(O)cc3OC2)c(OC)cc1O</smiles>

Figure 5. Miscellaneous chemical constituents from Erythrina species.

\section{Conclusion}

The chemical compounds from 15 species of Erythrina have been reviewed. Structurally unique along with many diversified compounds have been observed from this genus. Our study revealed that
Erythrina can be a prominent source of phytoconstituens as well as medicinal agents, and therefore other species of this genus need to be investigated for secondary metabolites. 


\section{References}

Amer, M.E., Shamma, M. and Freye, A.J. 1991. The tetracyclic Erythrina alkaloids. J. Nat. Prod. 54, 329363.

Amir, F., Yam, W.S. and Koay, Y.C, 2011. Phytochemical constituents and biological activities of Erythrina indica. European J. Chem. 2, 561-565.

Boland, G.M. and Donnelly, D.M.X. 1998. Isoflavonoids and related compounds. Nat. Prod. Rep. 241-260.

Chacha, M., Moleta, G.B. and Majinda, R.R.T. 2005. Antimicrobial and radical scavenging flavonoids from the stem wood of Erythrina latissima. Phytochemistry 66, 99-104.

Cui. L., Thuong, P.T., Fomum, Z.T. and Oh, W.K. 2009. A new erythrinan alkaloid from the seed of Erythrina addisoniae. Arch. Pharmacal. Res. 32, 325-328.

Faria, T.D.J., Cafeu, M.C., Akiyoshi, G., Ferreira, D.T., Galao, O.F., Andrei, C.C., Pinge-Filho, P., Paiva, M.R.C., Barbosa, A.D.M. and Braz-Filho, R. 2007. Alkaloids from flowers and leaves of Erythrina speciosa Andrews. Quimica Nova. 30, 525-527.

Folkers, K. and Major, R.T. 1937. Isolation of Erythroidine, an alkaloid of curare action from Erythrina americana Mill. J. Am. Chem. Soc. 59, 1580.

Garin-Aguilar, M.E., Ramirez-Luna, J.E., Soto-Hernandez, M., Valencia, D.T.G. and Martinez-Vazquez, M. 2000. Effect of crude extracts of Erythrina americana Mill. on aggressive behavior in rats. J. Ethnopharmacol. 69, 189.

Gillett, J.B. 1972. A further note on Erythrina melanacantha (Leguminosae-Papilionoideae), including a new subspecies from Somalia. Kew Bull. 27, 289-291.

Haggins, V. J. and Ingham, J.L. 1981. Demethylmedicaprin, a product formed from medicaprin by Colletotrichum coccodes. Phytopathology 71, 800-803.

Hauschild, W., Mutiso, P.B. and Passreiter, C.M., 2010. Prenylated pterocarpanes from Erythrina melanacantha. Nat. Prod. Commun. 5, 721-724.

Hussain, M.M., Dastagir, M.G., Billah, A.H.M.M. and Ismail, M. 2011. Alpinum isoflavone from Erythrina stricta Roxb. Bol. Latinoam. Caribe. Plant Med. Aromat. 10, 88-90.

Hussain, S.S., 2002. A new alkaloids from flowers of Erythrina stricta. J. Sci. I. R. Iran. 13, 35-38.

Hussain, M.M., Rahman, M.S., Jabbar, A. and Rashid, M.A. 2008. Phytochemical and biological investigation of Albizzia lebbeck. Bol. Latinoam. Caribe Plant Med. Aromat. 7, 273-278.

Innok, P., Rukachaisirikul, T., Phongpaichit, S. and Suksamrarn, A. 2010. Fuscacarpans A-C, new pterocarpans from the stems of Erythrina fusca. Fitoterapia. 81, 518-23.

Jang, J.P., Na, M.K., Thoung, P.T., Njamen, D., Mbafor, J.T., Fomum, Z.T., Woo, E.R. and Oh, W.K. 2008. Prenylated flavanoids with PTP1B inhibitory activity from the root bark of Erythrina mildbraedii. Chem. Pharm. Bull. 56, $85-88$.

Kabenei, J.S., Ndalut, P.K. and Sabah, A.O. 2011. Synergism of artemisinin with abyssinone-V from Erythrina abyssinica, (Lam. ex) against Plasmodium falciparum parasites: A potential anti-malarial combination therapy. J. Med. Plant Res. 5, 1355-1360.

Koo, J.Y., Oh, S., Cho, S.R., Koh, M., Oh, W.K., Choi, H.S. and Park, S.B. 2013. Total synthesis of Eryvarin H and its derivatives and their biological activity as ERR $\gamma$ inverse agonist. Org. Biomol. Chem. 11, 5782-5786.

Kobayashi, M., Mahmud, T., Yoshioka, N., Shibuya, H. and Kitagawa, I., 1997. Indonesian medicinal planst XXI. Inhibitors of $\mathrm{Na}^{+} / \mathrm{H}^{+}$exchanger from the bark of Erythrina variegata and the roots of Maclura cochinchinensis. Chem. Pharm. Bull. 45, 1615-1619.

Kumar, A., Lingadurai, S., Jain, A. and Barman, N.R. 2010. Erythrina variegata Linn: A review on morphology, phytochemistry, and pharmacological aspects. Pharmacog. Rev. 4, 147-152.

Lundquist, K. 1973. Acid degradation of Lignin. Acta Chem. Scan. 27, 2597-2606.

Miyazawa, M., Utsunomiya, H., Inada, K., Yamada, T., Okuno, Y., Tanaka, H. and Tatematsu, M. 2006. Inhibition of Helicobacter pylori motility by (+)syringaresinol from unripe Japanese apricot. Chem. Pharm. Bull. 29, 172-173.

Nakayama, M., Eguchi, S., Hayashi, S. and Tsukayama, M. 1978. The synthesis of neobavaisoflavone and related compounds. Chem. Pharm. Bull. 51, 2398-2400.

Nkengfack, A.E., Azebaze, A.G..B., Waffo, A.K., Fomum, Z.T., Meyer, M. and Heerden, F.R. 2001. Cytotoxic isoflavonoids from Erythrina indica Phytochemistry 58, 1113-1120.

Ozawa, M., Kishida, A. and Ohsaki, A. 2011. Erythrinan alkaloids from seeds of Erythrina velutina. Chem. Pharm. Bull. 59, 564-567.

Rahman, M.Z., Rahman, M.S., Kaisar, A., Hossain, A. and Rashid, M.A. 2010. Bioactive isoflavones from Erythrina variegata L. Turk. J. Pharm. Sci. 7, 21-28.

Rahman, M.Z, Sultana, S.J., Faroque, C.F., Ferdous, F., Rahman, M.S., Islam, M.S. and Rashid, M.A. 2007. Phytochemical and Biological investigation of Erythrina variegata. Saudi Pharm. J. 15, 140-145.

Rodriguez, R.R., Herrera, M.D., Perona, J.S. and Gutierrez, V.R. 2004. Potential vasorelaxant effects of oleanolic acid and erythrodiol two triterpenoids contained in "orujo" olive oil, on rat aorta. British J. Nut. 92, 635642.

Rukachaisirikul, T., Innok, P., Aroonrerk, N., Boonamnuaylap, W., Limrangsun, S., Boonyon, C., Woonjina, U. and Suksamrarn, A. 2007. Antibacterial pterocarpans from Erythrina subumbrans. J. Ethnopharmacol. 110, 171-175. 
Rukachaisirikul, T., Saekee, A., Tharibun, C., Watkuolham, S. and Suksamrarn, A. 2007. Biological activities of the chemical constituents of Erythrina stricta and Erythrina subumbrans. Arch. Pharmacal. Res. 30, 1398-1403.

Rukachaisirikul, T., Innok, P. and Suksamrarn, A. 2008. Erythrina alkaloids and a pterocarpens from the bark of Erythrina subumbrans. J. Nat. Prod. 71, 156-158.

Sato, M., Tanaka, H., Fujuwara, S., Hirata, M., Yamaguchi, R., Etoh, H. and Tokuda, C. 2003. Antibacterial property of isoflavanoids isolated from Erythrina variegata against cariogenic oral bacteria. Phytomedicine 10, 427433.

Soto-Hernandez M.R., Garcia-Mateos R., Migual-Chavez R.S. and Ramos-Valdivia C. 2012. Erythrina, a potential source of chemical from the Neotropics. Bioactive compounds in Phytomedicine, 163-184.

Talekepalli, H., Gollapudi, S.R., Shokri, A.K., Velazquez, L., Sandmann, R.A., Veliz, E.A., Rao, K.V.J., Madhavi, A.S. and Mitscher, L.A. 1990. Isoflavanoids and a cinnamyl phenol from root extracts of Erythrina variegata. Phytochemistry 29, 2005-2007.

Tanaka, H., Atsumi, I., Shirota, O., Sekita, S., Sakai, E., Sato, M., Murata, J., Murata, H., Darnaidi, D. and Chen, I.S. 2011. Three new constituents from the roots of Erythrina variegata and their antibacterial activity against methicillin-resistant Staphylococcus aureus. Chem. Biodiversity, 8, 476-482.

Tanaka, H., Hirata, M., Etoh, H., Sako, M., Sato, M., Murata, J., Murata, H., Dedy, D. and Fukai, T. 2004. Six new constituents from the roots of Erythrina variegata. Chem. Biodiversity 1, 1101-1108.

Tanaka, H., Tanaka, T. and Etoh, H. 1996. A pterocarpan from Erythrina orientalis. Phytochemistry 42, 14731475.

Tanaka, H., Etoh, H., Shimizu, H., Oh-Uchi, T., Terada, Y. and Tateishi, Y. 2001. Erythrinan alkaloids and isoflavonoids from Erythrina poeppigiana. Planta Med. 67, 871-873.

Tanaka, H., Hirata, M., Etoh, H., Shimizu, H., Sako, M., Murata, J., Murata, H., Darnaedi, D. and Fukai, T. 2003. Eryvarins $\mathrm{F}$ and $\mathrm{G}$, two 3-phenoxychromones from the roots of Erythrina variegata. Phytochemistry 62, 12431246.

Tanaka, H., Hirata, M., Etoh, H., Watanabe, N., Shimizu, H., Ahmad, M., Tareda, Y. and Fukai, T. 2002. Two diphenylpropan-1,2-diol syringates from the roots of Erythrina variegata. J. Nat. Prod. 65, 1933-1935.
Tanaka, H., Oh-Uchi, T., Etoh, H., Sako, M., Sudo, M., Sato, M., Fukai, T. and Tateishi, Y. 2003. An arylbenzofuran and four isoflavonoids from Erythrina poeppigiana. Phytochemistry 63, 597-602.

Tanaka, H., Sato, M., Fujiwara, S., Hirata, M., Etoh, H. and Takeuchi, H. 2002. Antibacterial activity of isoflavonoids isolated from Erythrina variegata against methicillin-resistant Staphylococcus aureus. Lett. App. Microbio. 35, 494-498.

Tanaka, H., Uchi, T.O., Etoh, H., Sako, M., Asai, F., Fukai, T., Sato, M., Murata, J. and Tbateishi, Y. 2003. Isoflavonoids from roots of Erythrina Zeyheri. Phytochemistry 64, 753-758.

Tchokouaha, R.F., Akexi, X., Chosson, E., Besson, T., Skaltsounis, A.L., Sequin, E., Alexis, M.N. and Wandji, J. 2010. Erymildbraedin A and B, two novel cytotoxic dimethylpyrano-isoflavones from the stem bark of Erythrina mildbraedii: evaluation of their activity toward endocrine cancer cells. J. Enzyme Inhib. Med. Chem. 25, 228-233.

Waffo, A.K., Azebaze, A.G..B., Nkengfack, A.E., Fomum, Z.T., Meyer, M., Bodo, B. and Heerden, F.R. 2000. Indicanines $\mathrm{B}$ and $\mathrm{C}$, two isoflavonoid derivatives from the root bark of Erythrina indica. Phytochemistry 53, 981-985.

Wanjala, C.C.W., Juma, B.F., Bojase, G., Gashe, B.A. and Majinda, R.R.T. 2002. Erythrinaline alkaloids and antimicrobial flavonoids from Erythrina latissima. Planta Med. 68, 640-642.

Watjen, W., Schnitker, A.K.S., Rohrig, R., Kulawik, A., Kyereme, J.A., Wright, C.W. and Passreiter, C.M. 2008. Prenylated flavonoids derivatives from the bark of Erythrina addisoniae. J. Nat. Prod. 71, 735-738.

Xiaoli, L., Naili, W., Sau, W.M., Chen, A.S.C. and Xinsheng, Y. 2006. Four new isoflavonoids from the stem bark of Erythrina variegata. Chem. Pharm. Bull. 54, 570-573.

Zheng, X., Wang, W., Piao, H., Xu, W., Shi, H. and Zhao, C. 2013. The genus Gnaphalium L. (Compositae): phytochemical and pharmacological characteristics. Molecules 18, 8298-8318.

Zhou, J., Xie, G. and Yan, X. 2011. Encyclopedia of Traditional Chinese Medicines. Vol. 2. 